\title{
Influência do método de síntese na obtenção de pós de $\mathrm{Na}_{2} \mathrm{TiSiO}_{5}$
}

\section{(Influence of the synthesis method to obtain $\mathrm{Na}_{2} \mathrm{TiSiO}_{5}$ powders)}

\author{
V. C. Albarici', M. T. Escote ${ }^{1}$, E. Orhan ${ }^{2}$, R. C. Lima ${ }^{1}$, J. A. Varela ${ }^{2}$, E. Longo ${ }^{1}$, E. R. Leite ${ }^{1}$ \\ ${ }^{1}$ CMDMC -LIEC, Departamento de Química, UFSCar \\ Rod. Washington Luiz, km 235, C.P. 676, S. Carlos, SP 13565-905 \\ ${ }^{2}$ CMDMC -LIEC, Instituto de Química, UNESP, Araraquara, SP 14801-907
}

\begin{abstract}
Resumo
Este trabalho apresenta um estudo sistemático sobre a síntese e caracterização de pós de $\mathrm{Na}_{2} \mathrm{TiSiO}_{5}$. Foram estudadas as propriedades estruturais e morfológicas dos pós por intermédio de difração de raios X, espectroscopia Raman e microscopia eletrônica de varredura. As amostras foram produzidas por meio de duas rotas sintéticas distintas baseadas em métodos tipo Pechini e submetidas a diferentes tratamentos térmicos. Medidas de espectroscopia Raman nas resinas poliméricas foram feitas visando verificar a influência das diferentes rotas utilizadas na síntese dos pós. Com os dados de difração de raios X foi feito um estudo da evolução das fases cristalográficas. A estrutura cristalina das amostras foi analisada por meio de refinamento Rietveld. Por fim, a morfologia dos compostos de $\mathrm{Na}_{2} \mathrm{TiSiO}_{5}$ foi examinada por microscopia eletrônica de varredura. Os resultados obtidos mostraram que é possível melhorar a qualidade das amostras de $\mathrm{Na}_{2} \mathrm{TiSiO}_{5}$ alterando-se a rota sintética utilizada.

Palavras-chave: síntese, Pechini, método de Rietveld.
\end{abstract}

\begin{abstract}
This work presents a systematic study of the synthesis and characterization of Na $\mathrm{TiSiO}_{5}$ compounds. We studied their structural and morphologic properties using X-ray diffraction, Raman spectroscopy and scanning electron microscopy. The samples were produced through two different synthetic routes, both based on Pechini method and heat treatments at different conditions. Raman spectroscopy measurements of the polymeric resins were performed in order to verify the influence of different routes on the obtained powders. The X-ray diffraction data were used to study the thermal evolution of the crystalline phases. The crystalline structures of the samples were analyzed by Rietveld refinement. Finally, the morphology of the $\mathrm{Na}_{2} \mathrm{TiSiO}_{5}$ compounds was investigated by scanning electron microscopy. Such results showed that the use of different synthetic routes allowed an improvement of the quality of $\mathrm{Na}_{2} \mathrm{TiSiO}_{5}$ samples.

Keywords: synthesis, Pechini, Rietveld method.
\end{abstract}

\section{INTRODUÇÃO}

$\mathrm{O}$ titanossilicato de sódio é um material de fórmula geral $\mathrm{A}_{2} \mathrm{TiBO}_{5}(\mathrm{~A}=\mathrm{Na}$ ou $\mathrm{Li}$ e $\mathrm{B}=\mathrm{Si}$ ou $\mathrm{Ge})$ e apresenta em sua estrutura cristalina centros de titânio penta coordenados. A descoberta de luminescência à temperatura ambiente nestes materiais torna os compostos desta família interessantes de serem estudados [1].

$\mathrm{O} \mathrm{Na}_{2} \mathrm{TiSiO}_{5}$ apresenta trimorfismo e pode ser obtido em sua fase tetragonal a altas temperaturas $\left(\mathrm{HT}-\mathrm{Na}_{2} \mathrm{TiSiO}_{5}\right)$, em sua fase ortorrômbica formada em baixas temperaturas (LT$\mathrm{Na}_{2} \mathrm{TiSiO}_{5}$ ), e a chamada natisita que pode ser obtida sob condições hidrotermais [2]. O LT-Na $\mathrm{TiSiO}_{5}$ cristaliza-se em uma estrutura ortorrômbica pertencente ao grupo espacial Pmc21 [2].

Particularmente, estudos realizados acerca da família de compostos $\mathrm{A}_{2} \mathrm{TiBO}_{5}$ revelam as dificuldades na obtenção de amostras monofásicas. Entre os métodos utilizados estão o método convencional de mistura de óxidos [2] e hidrotermal [3].
No entanto, não foram encontrados materiais deste tipo obtidos por métodos tipo Pechini, o que justifica o estudo da síntese dos mesmos por intermédio deste tipo de rota. Além disso, estudos baseados na preparação de materiais cerâmicos utilizando o método proposto por Pechini [4] têm mostrado a possibilidade de obter titanatos [5-8] e também materiais contendo $\mathrm{SiO}_{2}[9$, $10]$ com boas qualidades cristalográficas e físicas.

O método Pechini (MP) original consiste na síntese de um poliéster a partir de uma reação de condensação de um poliálcool, geralmente etilenoglicol (EG), com um ácido carboxílico como, por exemplo, ácido cítrico (AC). Os cátions metálicos são então solubilizados nesta matriz polimérica segundo a estequiometria desejada.

Este método, no entanto, apresenta algumas limitações, devido à solubilidade dos cátions metálicos na matriz polimérica e, também, devido à reação de condensação entre $\mathrm{AC}$ e EG, que pode ocorrer em qualquer carboxila do $\mathrm{AC}$ sendo difícil a previsão de processos como aumento da cadeia polimérica [11]. 
Com a finalidade de solucionar estas limitações descritas foram desenvolvidas variações deste método. Uma das variações do MP é o método de polimerização de complexos (MPC), que assim como MP [4] utiliza a polimerização "in situ”, ou seja, a polimerização ocorre no interior do próprio vaso reacional. No entanto, a diferença entre estes métodos está basicamente na maneira de preparação da resina [12]. $\mathrm{Na}$ rota via MPC são preparados compostos de coordenação metálicos solúveis com $\mathrm{AC}$, e só então é adicionado o poliálcool EG, que promove a polimerização dos complexos metálicos, o que caracteriza o nome dado ao método.

No presente trabalho estudou-se a síntese de pós de $\mathrm{Na}_{2} \mathrm{TiSiO}_{5}$ por intermédio das rotas baseadas no MP e MPC e estudou-se a influência da rota sintética nas propriedades físicas das amostras. Isto foi feito por meio de caracterizações por espectroscopia Raman, difração de raios X (DRX) e microscopia eletrônica de varredura (MEV).

\section{MATERIAIS E MÉTODOS}

\section{Preparação dos Pós de $\mathrm{Na}_{2} \mathrm{TiSiO}_{5}$}

As resinas poliméricas de $\mathrm{Na}_{2} \mathrm{TiSiO}_{5}$ foram obtidas por intermédio das rotas sintéticas 1 e 2 , de modo que a rota sintética denominada 1 é baseada no método Pechini original, onde a matriz polimérica é obtida seguida da solubilização dos cátions na mesma. Já a rota sintética denominada 2 é baseada no método de polimerização de complexos, no qual inicialmente são formados os compostos de coordenação e somente então é promovida a polimerização. A principal diferença entre as rotas

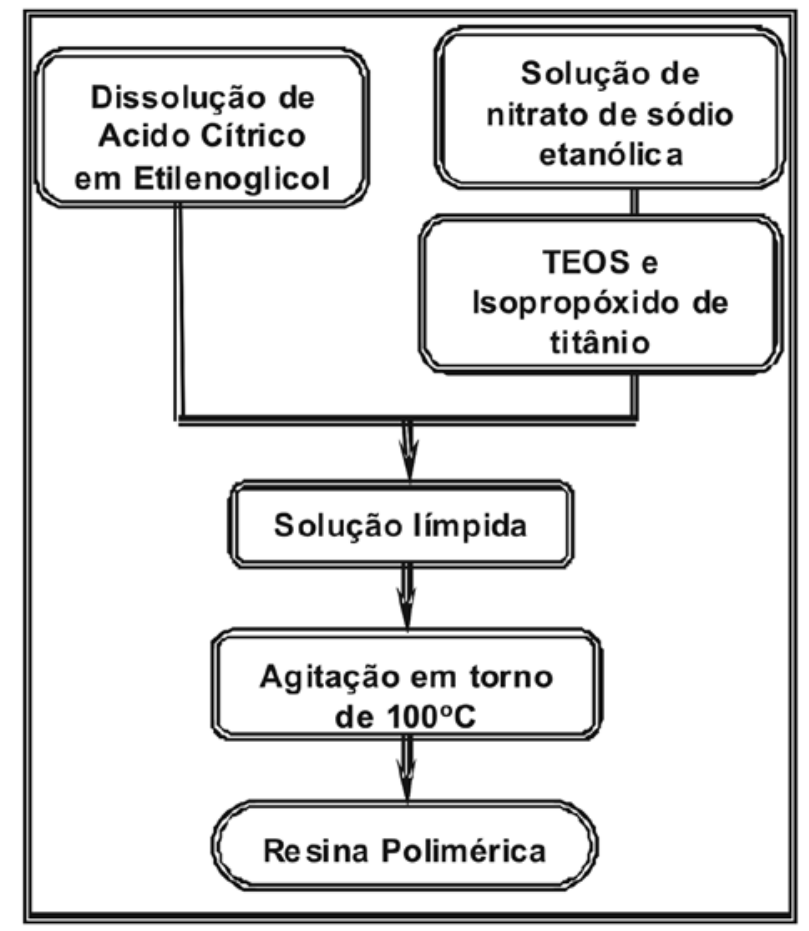

Figura 1: Fluxograma referente à rota sintética 1. [Figure 1: Flow chart illustrating the synthetic route 1.]

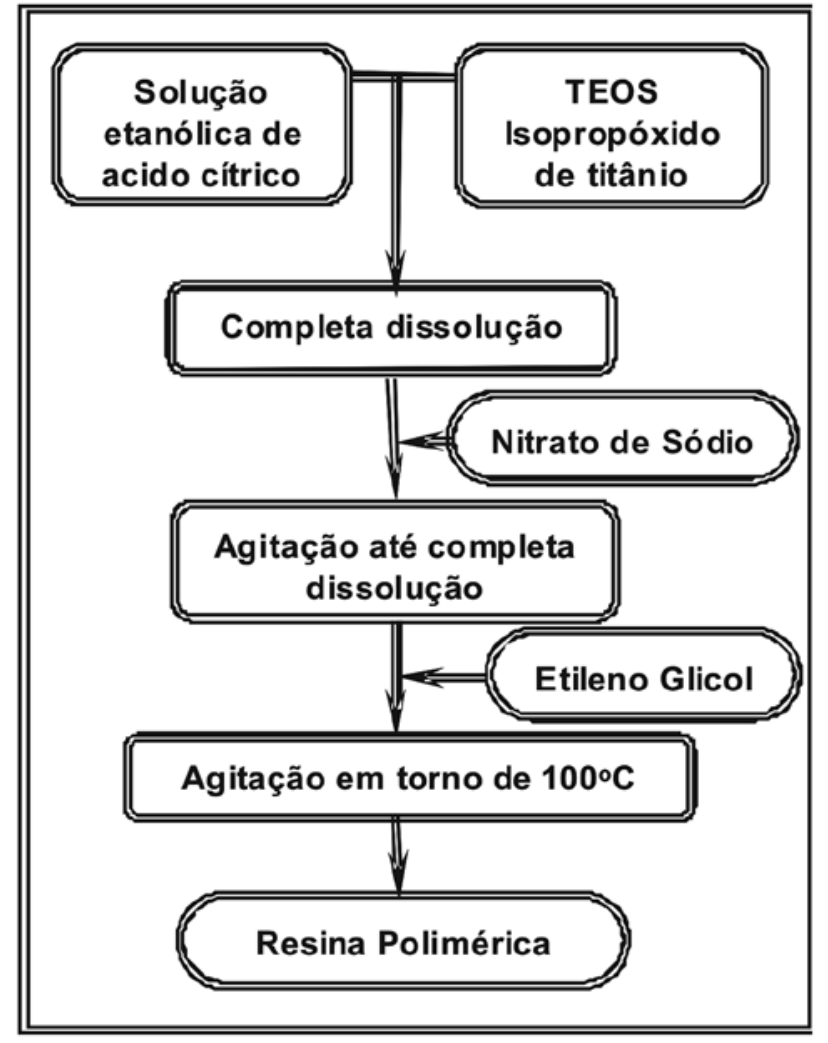

Figura 2: Fluxograma referente à rota sintética 2.

[Figure 2: Flow chart illustrating the synthetic route 2.]

é o momento de adição do etilenoglicol. As Figs. 1 e 2 ilustram os fluxogramas das rotas sintéticas 1 e 2 respectivamente.

Para um melhor entendimento, foram utilizados códigos para as amostras obtidas, sendo denominadas de NTS1 e NTS2 que correspondem às amostras sintetizadas pelas rotas 1 e 2 , respectivamente. As soluções precursoras foram preparadas em etanol anidro, utilizando-se ácido cítrico como agente quelante e etilenoglicol para que ocorresse uma reação de esterificação. A polimerização, promovida pelo aquecimento da mistura resultou em uma solução homogênea, na qual os cátions metálicos estão uniformemente distribuídos na matriz orgânica. Esta reação ocorre quando o solvente é eliminado em um intervalo de temperatura de $90{ }^{\circ} \mathrm{C}-120{ }^{\circ} \mathrm{C}$. Os precursores poliméricos foram calcinados a $400{ }^{\circ} \mathrm{C}$ para pirólise, durante a qual ocorreu a eliminação de material orgânico e etanol, obtendo-se então um carvão. Em seguida, os materiais obtidos foram submetidos a diferentes tratamentos térmicos à temperatura de 600, 700 e $800{ }^{\circ} \mathrm{C}$ durante $4 \mathrm{~h}$. Por fim, estes pós foram submetidos a tratamentos em 800 e $850^{\circ} \mathrm{C}$ durante $24 \mathrm{~h}$. Como fonte de titânio silício e sódio utilizou-se o isopropóxido de titânio, o tetra etil ortosilicato (TEOS) e nitrato de sódio respectivamente.

\section{Técnicas de caracterização}

As resinas poliméricas bem como alguns precursores usados na síntese foram analisados por espectroscopia 
Raman, com o intuito de investigar as diferenças entre as duas rotas usadas. Para a espectroscopia Raman, foi utilizado um espectrofotômetro Bruker RFS100, equipado com um laser Nd-YAG $(\lambda=1064 \mathrm{~nm})$, usando uma potência de 23 $\mathrm{mW}$, com 500 varreduras e resolução de $4 \mathrm{~cm}^{-1}$.

As amostras sintetizadas foram analisadas por DRX, que permitiu avaliar as fases cristalográficas apresentadas pelas amostras. O equipamento utilizado para a análise de DRX foi um difratômetro Rigaku anodo rotatório. As condições usadas para as análises de rotina foram: $2 \theta$ variando de 5 a $75^{\circ}$, tempo de exposição de 1,0 s e passo angular de $0,030^{\circ}$, radiação $\mathrm{Cu}-\mathrm{K} \alpha$ e para a obtenção dos difratogramas para análise por refinamento Rietveld foram: $2 \theta$ variando de 15 a $85^{\circ}$, tempo de exposição de 8,0 s e passo angular de $0,020^{\circ}$, radiação $\mathrm{CuK} \alpha$. As análises por método Rietveld foram feitas nos padrões de difração de raios $\mathrm{X}$ das amostras calcinadas a $850{ }^{\circ} \mathrm{C}$ e que forneceram os parâmetros estruturais do composto $\mathrm{Na}_{2} \mathrm{TiSiO}_{5}$ e permitiram identificar e quantificar as fases presentes nestas amostras. O programa utilizado para o refinamento Rietveld foi o Fullproof 2002 para microcomputador [13]. Para esta análise foram utilizados alguns parâmetros estruturais iniciais como: número de fases cristalinas, grupo espacial, parâmetros de rede, posições atômicas, de cada fase existente na amostra, estes dados utilizados estão listados nas referências [3, 14]. O tipo de função utilizada para o ajuste foi a pseudo-Voigt.

As análises por MEV foram realizadas utilizando um microscópio eletrônico Zeiss modelo DSM940A. As micrografias foram obtidas utilizando-se um potencial de $20 \mathrm{kV}$ e corrente de $80 \mathrm{~mA}$. As imagens foram geradas por elétrons secundários possibilitando uma análise topográfica das amostras, sendo possível verificar sua morfologia.

\section{RESULTADOS E DISCUSSÃO}

Nesta seção serão apresentados inicialmente os dados de espectroscopia Raman das resinas precursoras dos compostos de NTS e alguns precursores utilizados para a preparação das resinas. Em seguida, serão mostrados os resultados e análises realizadas nas caracterizações por meio de DRX, refinamentos pelo método de Rietveld e MEV. Estes serão descritos com mais detalhes a seguir.

\section{Espectroscopia Raman das resinas}

Primeiramente foram realizados experimentos de espectroscopia Raman nas resinas poliméricas e alguns precursores utilizados com a finalidade de compreender as etapas de formação dos complexos em cada uma das rotas sintéticas utilizadas. Para isso, foram obtidos espectros de FTRaman para o EG, AC e do EG + AC. Devido à semelhança entre os resultados encontrados para as resinas poliméricas obtidas pelas rotas 1 e 2 optou-se por apresentar somente o espectro Raman das resinas de NTS obtidas por intermédio da rota 1. Estes resultados são apresentados na Fig 3.

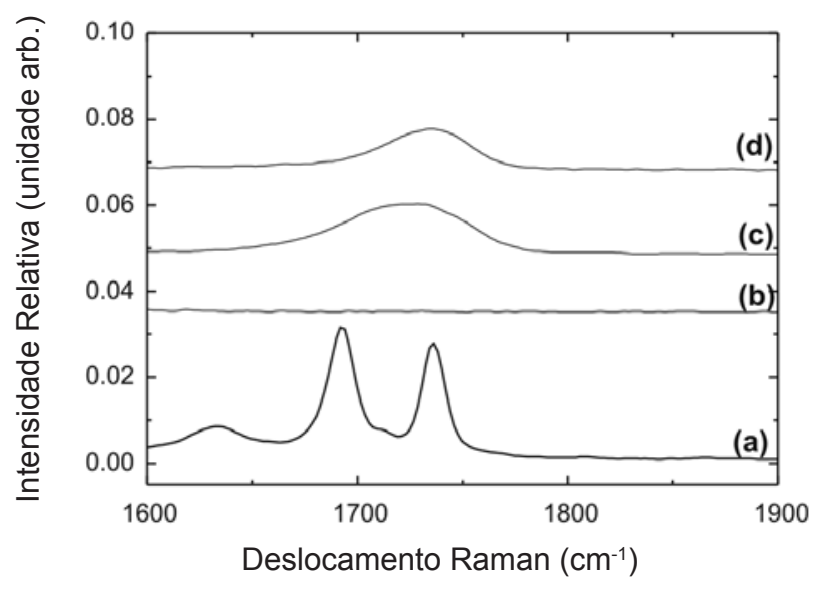

Figura 3: Espectros Raman para (a) AC; (b) EG; (c) AC+EG; (d) Resina polimérica NTS1.

[Figure 3: Raman spectra for (a) $A C$; (b) $E G$; (c) $A C+E G$; (d) Polymeric resin NTS1.]

Na Fig. 3(a) pode-se verificar a ocorrência de três picos distintos na região de $1600-1800 \mathrm{~cm}^{-1}$ que foram identificados como pertencentes às carboxilas livres do AC. Quando comparado com o espectro (b), verifica-se na mesma região a formação de uma única banda larga. Este resultado sugere que quando o AC é adicionado ao EG as carboxilas livres do AC são afetadas, o que pode resultar então em uma concorrência entre os cátions metálicos e o EG durante a complexação dos metais [15]. No caso do espectro Raman obtidos para a resina de NTS1 verifica-se um comportamento semelhante ao mostrado para o $\mathrm{AC}+\mathrm{EG}$, o que indica que as carboxilas do AC foram consumidas tanto pela complexação quanto pela polimerização. Os resultados acima mencionados sugerem que a rota sintética denominada rota 2 tende a dificultar a segregação de fases, já que a complexação dos cátions pelas carboxilas do AC ocorre sem a presença da concorrência do EG.

\section{Análises por difração de raios $X$}

As resinas poliméricas obtidas por intermédio das rotas 1 e 2 foram submetidas a diferentes tratamentos térmicos e a evolução térmica dos compostos obtidos foi analisada por difração de raios $\mathrm{X}$. Os difratogramas das amostras calcinadas a $600,700,800{ }^{\circ} \mathrm{C}$ durante $4 \mathrm{~h}$ e $850{ }^{\circ} \mathrm{C}$ durante $24 \mathrm{~h}$ são apresentadas na Figs. 4 e 5, respectivamente. Nestas figuras pode-se verificar que tanto o difratograma da amostra de NTS1 quanto da NTS2 calcinadas a $600{ }^{\circ} \mathrm{C}$ apresentaram alguns picos que caracterizam o inicio do processo de cristalização. A maioria destes picos foi identificada como pertencentes à fase cristalina desejada de $\mathrm{Na}_{2} \mathrm{TiSiO}_{5}$. A partir do tratamento térmico a $700{ }^{\circ} \mathrm{C}$, as amostras apresentam um alto grau de cristalinidade caracterizado pelo claro estreitamento das reflexões de Bragg, além disso, a partir desta temperatura foram identificados picos de fases adicionais pertencentes à fase deletéria de $\mathrm{Na}_{2} \mathrm{Ti}_{6} \mathrm{O}_{13}(\mathrm{~T})$. 


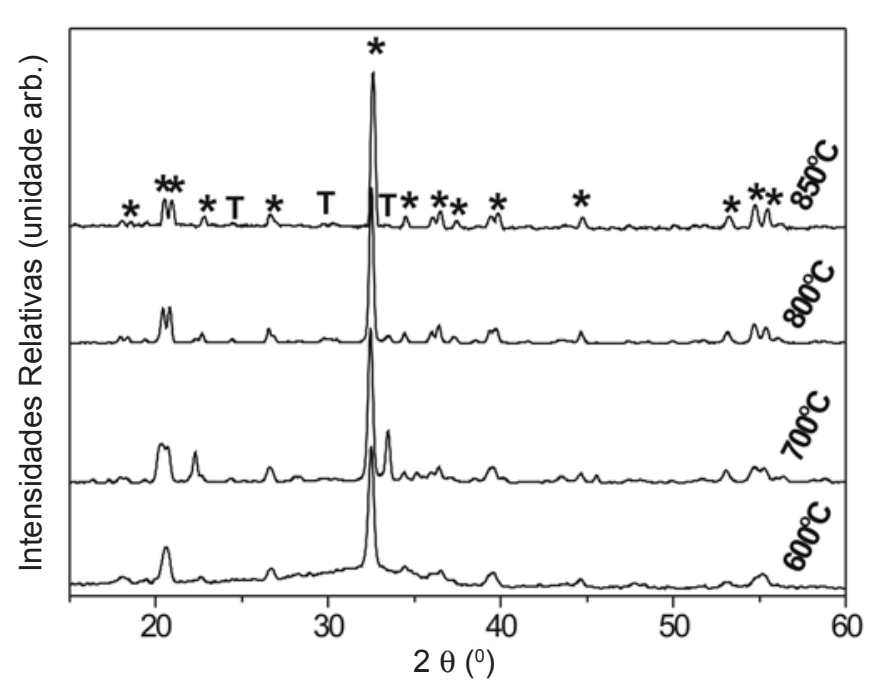

Figura 4: Difratogramas de raios X para as amostras de NTS1 tratados termicamente em diferentes temperaturas. Os símbolos $*$ e T correspondem às reflexões do $\mathrm{Na}_{2} \mathrm{TiSiO}_{5}$ e $\mathrm{Na}_{2} \mathrm{Ti}_{6} \mathrm{O}_{13}$, respectivamente.

[Figure 4: X-ray diffraction patterns of NTS1 samples annealed at different temperatures. The symbols $*$ and $\mathrm{T}$ indicate the $\mathrm{Na}_{2} \mathrm{TiSiO}_{5}$ and $\mathrm{Na}_{2} \mathrm{Ti}_{6} \mathrm{O}_{13}$ reflections, respectively.]

A fim de se obter amostras de NTS1 e NTS2 com menores proporções de fases adicionais foram feitos tratamentos térmicos variando-se a temperatura entre 800 e $850{ }^{\circ} \mathrm{C}$ e o tempo do patamar até $24 \mathrm{~h}$. É importante relatar que a temperatura de $850{ }^{\circ} \mathrm{C}$ foi a máxima atingida antes do início da fusão do material. Pode-se perceber, fazendo-se uma comparação entre estas figuras, que as intensidades relativas dos picos de fases adicionais decrescem com o aumento da temperatura e o tempo de calcinação, o que sugere que as quantidades destas fases também estejam diminuindo.

Para verificar este ponto, assim como estudar a estrutura cristalina das amostras de NTS1 e NTS2, os dados de difração de raios $\mathrm{X}$ dos pós tratados termicamente a $850{ }^{\circ} \mathrm{C}$ foram analisados por meio de refinamento Rietveld, como descrito a seguir.

\section{Refinamentos pelo método de Rietveld}

Os difratogramas das amostras de NTS1 e NTS2 calcinadas a $850{ }^{\circ} \mathrm{C}$ durante $24 \mathrm{~h}$ foram analisados por intermédio de refinamentos pelo método de Rietveld utilizando-se o programa Fullproof. Os parâmetros iniciais utilizados para o refinamento estrutural destas amostras serão apresentados juntamente com os resultados do refinamento para comparação. Como descrito na literatura o composto $\mathrm{Na}_{2} \mathrm{TiSiO}_{5}$ cristaliza-se em uma estrutura ortorrômbica e na simetria $\mathrm{Pmc} 2_{1}$ [3]. Os principais parâmetros refinados foram: o fator de escala, o "background", os parâmetros de rede, a forma e as larguras dos picos, os fatores térmicos e as posições atômicas.

Estes refinamentos foram realizados para as amostras de NTS1 e NTS2, e a Fig. 6 apresenta um exemplo do gráfico

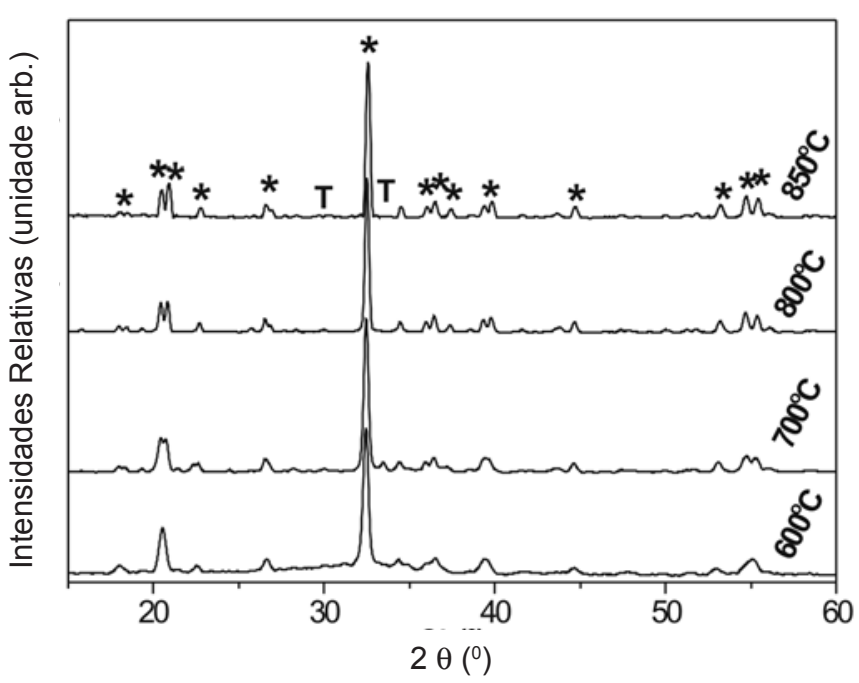

Figura 5: Difratogramas de raios X para as amostras de NTS2 tratadas termicamente em diferentes temperaturas, onde $*=\mathrm{Na}_{2} \mathrm{TiSiO}_{5}$, $\mathrm{T}=\mathrm{Na}_{2} \mathrm{Ti}_{6} \mathrm{O}_{13}$.

[Figure 5: X-ray diffraction patterns of NTS2 samples annealed at different temperatures, where $*=\mathrm{Na}_{2} \mathrm{TiSiO}_{5}, \mathrm{~T}=\mathrm{Na}_{2} \mathrm{Ti}_{6} \mathrm{O}_{13}$.]

representando o refinamento para a amostra NTS2. A figura apresenta o padrão de difração calculado pelo programa (linha preta), o obtido experimentalmente (círculos vazados) e a diferença entre os dois (linha preta) abaixo dos difratogramas.

Os coeficientes de confiabilidade Rwp, Rp, S e $\mathrm{R}_{\text {Bragg, }}$, que são os indicadores de qualidade do refinamento, estão listados na Tabela I. Os valores de $\mathrm{S}$ próximos de 1 e $\mathrm{R}_{\mathrm{Bragg}}$ aproximadamente 3 caracterizam um bom ajuste dos padrões de difração de raios $\mathrm{X}$. Os valores de $\mathrm{R}_{\text {Bragg }}$ encontrados para as fases adicionais foram da ordem de 11,5 para a rota $1 \mathrm{e}$ 9,69 para a rota 2 . Acredita-se que estes valores elevados estejam relacionados ao fato das reflexões pertencentes às fases adicionais serem pouco intensas.

O refinamento também permitiu a quantificação da fase principal de NTS, bem como das fases secundárias identificadas. A Tabela I mostra as porcentagens (em massa) de fases presentes nas amostras NTS1 e NTS2 e estimadas por intermédio de refinamento Rietiveld. Nesta tabela verifica-se que a amostra NTS2 apresentou uma quantidade de fase adicional consideravelmente menor $(\sim 60 \%)$ do que aquela apresentada pela amostra NTS1.

Com base nas análises quantitativas de fases podese inferir que a amostra de NTS2 apresentou menores quantidades de fases secundárias, sendo assim mais indicada para a síntese destes materiais. É importante ressaltar que estes resultados estão de acordo com a literatura onde os pós de $\mathrm{Na}_{2} \mathrm{TiSiO}_{5}$ obtidos não são monofásicos, ou seja, apresentam fases adicionais $[2,3]$.

Os principais parâmetros obtidos no refinamento de Rietveld para o NTS1 e NTS2, bem como resultados reportados por ZIADI et al [3], são apresentados a seguir, 
sendo que os parâmetros de rede $a, b$ e $c$ e o volume da célula unitária podem ser vistos na Tabela I. Os valores de $b$ e $c$ foram ligeiramente maiores e o valor de a menor do que aqueles listados na literatura (veja Tabela I). Entretanto, de modo geral, os parâmetros refinados mostram-se próximos àqueles listados e reportados na referência [3].

\section{Microscopia Eletrônica de Varredura}

A partir dos resultados acima apresentados, foram selecionadas algumas amostras que foram analisadas por meio de MEV. Isto foi feito com o intuito de obter-se informações acerca da morfologia e tamanho dos grãos das mesmas.

Estas análises combinadas com a difração de raios $\mathrm{X}$ podem contribuir com importantes informações para $\mathrm{O}$ trabalho. Neste caso, para o estudo da morfologia durante a evolução térmica foram analisadas por microscopia apenas as amostras sintetizadas pela rota 2. Assim sendo, foram feitas imagens de MEV para as amostras de NTS2 obtidas a 600 e $700{ }^{\circ} \mathrm{C}$ por $4 \mathrm{~h}$ bem como a $850{ }^{\circ} \mathrm{C}$ por $24 \mathrm{~h}$. Para a
Tabela I - Indicadores dos refinamentos, proporções das fases encontradas, parâmetros de rede e volume da célula unitária para as amostras NTS1 e NTS2.

[Table I- The refinement indicators, phase proportions, lattice parameters and unit cell volume for the NTS1 e NTS2 samples.]

\begin{tabular}{cccc}
\hline & Referência* & NTS1 & NTS2 \\
\hline $\mathrm{Rwp} \%$ & - & 10,5 & 9,45 \\
$\mathrm{R} e x p$ & - & 9,18 & 7,80 \\
$\mathrm{~S}$ & - & 1,44 & 1,21 \\
$\mathrm{R}_{\mathrm{Bragg}}$ & - & 4,84 & 3,93 \\
$\% \mathrm{Na}_{2} \mathrm{TiSiO}_{5}$ & - & $93,8(7)$ & $96,4(7)$ \\
$\% \mathrm{Na}_{2} \mathrm{Ti}_{6} \mathrm{O}_{13}$ & - & $6,25(9)$ & $3,72(9)$ \\
$\mathrm{a}(\AA)$ & $9,1814(3)$ & $9,158(4)$ & $9,158(4)$ \\
$\mathrm{b}(\AA)$ & $4,8075(2)$ & $4,818(4)$ & $4,814(4)$ \\
$\mathrm{c}(\AA)$ & $9,8604(3)$ & $9,875(2)$ & $9,869(2)$ \\
$\mathrm{V}\left(\AA^{3}\right)$ & $435,23(3)$ & $435,71(2)$ & $435,13(2)$ \\
\hline
\end{tabular}

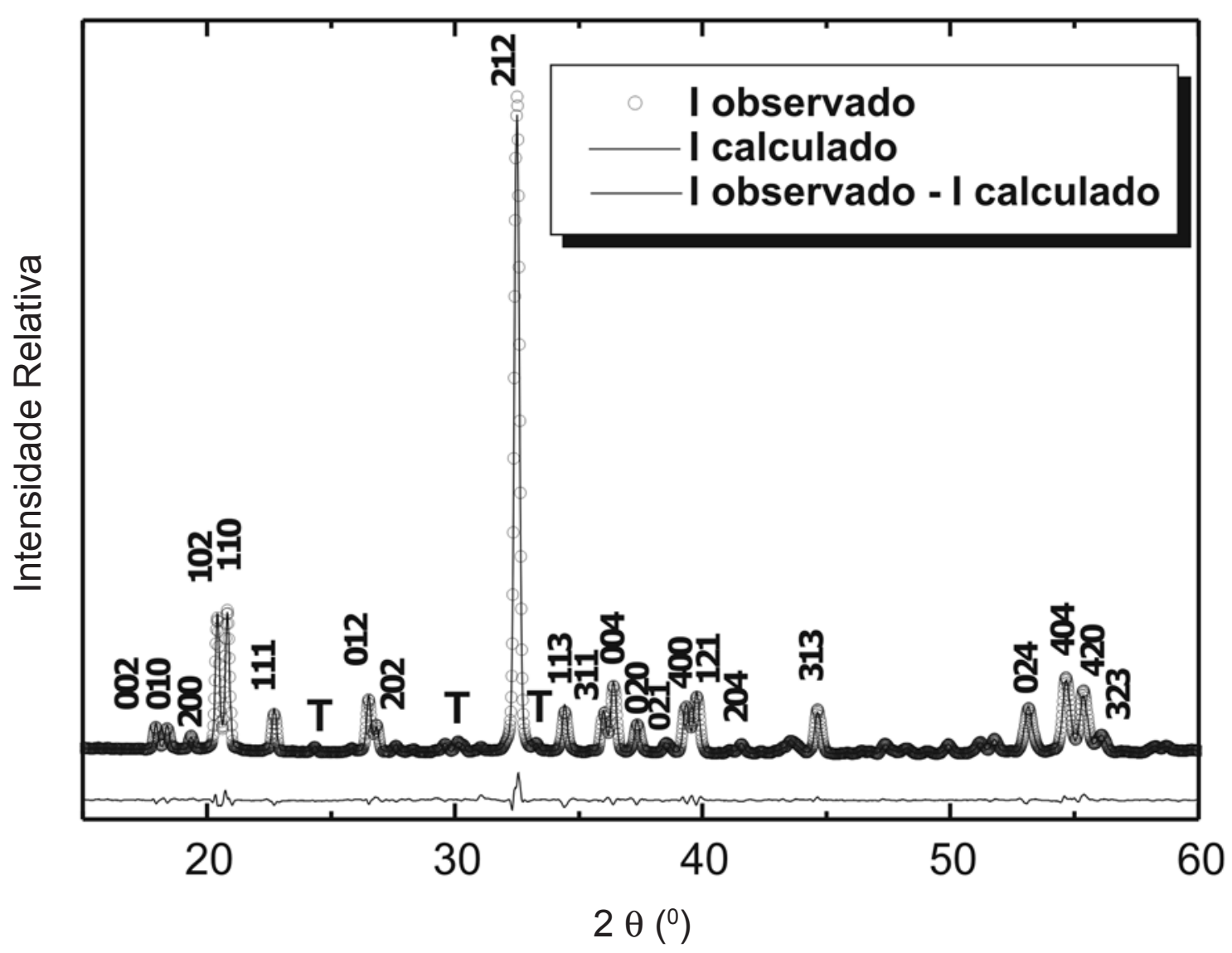

Figura 6: Difratograma de raios X observado experimentalmente e calculado pelo refinamento de Rietveld do pó de NTS2, onde NTS indica a fase de $\mathrm{Na}_{2} \mathrm{TiSiO}_{5}$ e $\mathrm{T}$ a fase de $\mathrm{Na}_{2} \mathrm{Ti}_{6} \mathrm{O}_{13}$.

[Figure 6: Experimental and calculated XRD patterns obtained by Rietveld refinement for the NTS2 powder, where NTS indicated the $\mathrm{Na}_{2} \mathrm{TiSiO}_{5}$ phase and $\mathrm{T}$ the $\mathrm{Na}_{2} \mathrm{Ti}_{6} \mathrm{O}_{13}$ phase.] 

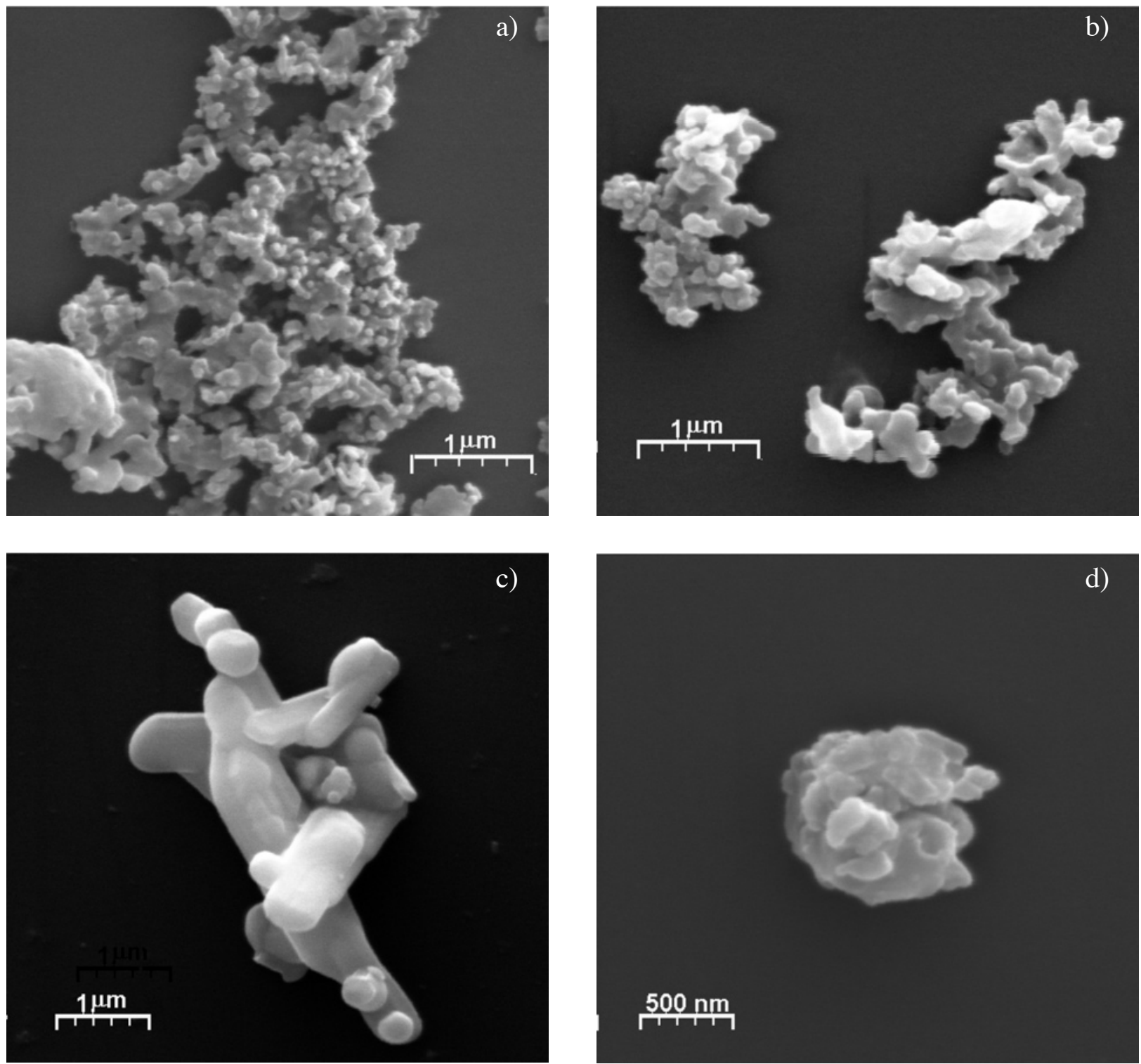

Figura 7: Micrografias de MEV das amostras: (a) NTS2 $600^{\circ} \mathrm{C}$ com aumento de $20000 \mathrm{x}$; (b) NTS2 $700{ }^{\circ} \mathrm{C}$ com aumento de 20000 $\mathrm{x}$; (c) NTS2 $850^{\circ} \mathrm{C}$ com aumento de $15000 \mathrm{x}$; (d) NTS1 $850^{\circ} \mathrm{C}$ com aumento de $30000 \mathrm{x}$.

[Figure 7: SEM images of samples: (a) NTS2 $600{ }^{\circ} \mathrm{C}$ with magnification of 20000x; (b) NTS2 $700{ }^{\circ} \mathrm{C}$ with magnification of 20000x; (c) NTS2 $850^{\circ} \mathrm{C}$ with magnification of 15000x; (d) NTS1 $850^{\circ} \mathrm{C}$ with magnification of 30000x.]

comparação da morfologia das amostras cristalinas obtidas pelas duas rotas sintéticas distintas, a amostra de NTS1 calcinada a $850{ }^{\circ} \mathrm{C}$ durante $24 \mathrm{~h}$ também foi analisada. As micrografias destes materiais são apresentadas na Fig. 7.

Uma inspeção cuidadosa destas micrografias revela que todas as amostras de NTS sintetizadas estão aglomeradas. Entretanto, é importante ressaltar que isto é característico do método de síntese tipo Pechini, como foi observado por Leite et al. [16]. Ao analisar-se cada amostra particularmente tem-se que: em (a) a presença de grãos com morfologias arredondados com um certo alongamento. Em (b) tem-se o alongamento dos grãos mais evidente indicando também coalescência entre os mesmos. Nas Figs. 7 (c) e (d) verifica- se sinais de coalescência mais evidentes. De modo geral, verifica-se um aumento no tamanho das partículas com o aumento da temperatura.

\section{CONCLUSÕES}

Com base nos resultados obtidos neste trabalho, pode-se concluir que os resultados de espectroscopia Raman sugerem que a concorrência entre os cátions e o EG durante a complexação pode ser associado ao fato das amostras obtidas por intermédio da rota sintética 1 apresentarem maior quantidade de fases deletérias. Isto devido a esta análise indicar a ocorrência de sítios de 
complexação já polimerizados pelo etilenoglicol, antes mesmo da adição dos cátions metálicos quando esta rota é utilizada.

Os métodos tipo Pechini utilizados neste trabalho mostraram-se eficientes para a obtenção da fase cristalina de $\mathrm{Na}_{2} \mathrm{TiSiO}_{5}$ e, de modo geral, as estruturas cristalinas das amostras estão de acordo com aquelas apresentadas na literatura. Entretanto, os resultados obtidos mostraram que a rota sintética 2 é mais indicada para tanto, pois permitiu a produção de amostras de $\mathrm{Na}_{2} \mathrm{TiSiO}_{5}$ com menores quantidades de fases adicionais.

\section{REFERÊNCIAS}

[1] B. Bouma, G. Blasse, J. Phys. Chem. Solids 56, 2 (1995) 261.

[2] A. Ziadi, H. Hillebrecht, G. Thiele, B. Elouadi, J. Solid State Chem. 123 (1996) 324.

[3] G. W. Peng, H. S.Liu, Mat. Chem. Phys. 42, 4 (1995) 264.

[4] M. P. Pechini, U. S. Patent 3330697 (1967).

[5] F. M. Pontes, E. B. Araújo, E. R. Leite, J. A. Eiras, E. Longo, J. A. Varela, M. A. Pereira-da-Silva, J. Mater. Res. 15, 5 (2000) 1176.
[6] E. J. H. Lee , F. M. Pontes, E. R. Leite, E. Longo, R. Magnani, P. S. Pizani, J. A. Varela, Mater. Lett. 58, 11 (2004) 1715 .

[7] E. C. Paris, E. R. Leite, E. Longo, J. A. Varela, Mater. Lett. 37 (1998) 1.

[8] F. M. Pontes, E. Longo, J. H. G. Rangel, M. I. Bernardi, E. R. Leite, J. A. Varela, Mater. Lett. 43 (200) 249.

[9] E. R.Leite, N. L. V. Carreño, E. Longo, F. M. Pontes, A. Barison, A. G. Ferreira, Y. Maniette, J. A. Varela, Chem. Mater. 14 (2002) 3722.

[10] N. L. V. Carreño, E. R. Leite, L. P. S. Santos, P. N. Lsboa-Filho, E. Longo, G. C. L. Araujo, A. Barison, A. G. Ferreira, A. Valentini, L. F. D. Probst, Quim. Nova 25, 6 (2002) 935.

[11] M. Kakihana, J. Sol-Gel Sci. Tech. 6 (1996) 5.

[12] T. Okubo, M. Kakihana, J. Alloy Comp. 256 (1997) 151.

[13] J. Rodriguez-Carvajal, Physica B: Condensed Matter. 192, 1-2 (1993) 55.

[14] S. Andersson, A. D. Wadsley, Acta Cryst. 15 , 3 (1962) 194.

[15] M. Kakihana, M. Yoshimura, Bull. Chem. Soc. Jpn. 72, 7 (1999) 1427.

[16] E. R. Leite, M. A. L. Nobre, M. Cerqueira, E. Longo, J. A. Varela, J. Am. Ceram. Soc. 80 (1997) 2649. 